\title{
Toward a Lingua Franca for Deterministic Concurrent Systems
}

MARTEN LOHSTROH, University of California, Berkeley, USA

CHRISTIAN MENARD, TU Dresden, Germany

SOROUSH BATENI, University of Texas at Dallas, USA

EDWARD A. LEE, University of California, Berkeley, USA

Many programming languages and programming frameworks focus on parallel and distributed computing. Several frameworks are based on actors, which provide a more disciplined model for concurrency than threads. The interactions between actors, however, if not constrained, admit nondeterminism. As a consequence, actor programs may exhibit unintended behaviors and are less amenable to rigorous testing. We show that nondeterminism can be handled in a number of ways, surveying dataflow dialects, process networks, synchronous-reactive models, and discrete-event models. These existing approaches, however, tend to require centralized control, pose challenges to modular system design, or introduce a single point of failure. We describe "reactors," a new coordination model that combines ideas from several of these approaches to enable determinism while preserving much of the style of actors. Reactors promote modularity and allow for distributed execution. By using a logical model of time that can be associated with physical time, reactors also provide control over timing. Reactors also expose parallelism that can be exploited on multicore machines and in distributed configurations without compromising determinacy.

CCS Concepts: • Computing methodologies $\rightarrow$ Concurrent programming languages; $\bullet$ Software and its engineering $\rightarrow$ Source code generation; Runtime environments; • Computer systems organization $\rightarrow$ Real-time languages;

Additional Key Words and Phrases: Polyglot, coordination language, concurrency, determinism

\section{ACM Reference format:}

Marten Lohstroh, Christian Menard, Soroush Bateni, and Edward A. Lee. 2021. Toward a Lingua Franca for Deterministic Concurrent Systems. ACM Trans. Embed. Comput. Syst. 20, 4, Article 36 (May 2021), 27 pages. https://doi.org/10.1145/3448128

\section{INTRODUCTION}

Loosely, actors are concurrent objects that communicate by sending each other messages. Under this definition, a large number of actor programming languages and models have been developed,

The work in this article was supported in part by the National Science Foundation (NSF), award \# CNS-1836601 (Reconciling Safety with the Internet) and the iCyPhy (Industrial Cyber-Physical Systems) research center, supported by Denso, Siemens, and Toyota.

Authors' addresses: M. Lohstroh and E. A. Lee, University of California, Berkeley, 545Q Cory Hall, Berkeley, CA, 94720; emails: \{marten, eal\}@berkeley.edu; C. Menard, TU Dresden, Chair for Compiler Construction, Georg-Schumann-Str. 11, 01069, Dresden, Germany; email: christian.menard@tu-dresden.de; S. Bateni, University of Texas at Dallas, 800 W. Campbell Road, Richardson, TX, 75080; email: soroush@utdallas.edu.

Permission to make digital or hard copies of part or all of this work for personal or classroom use is granted without fee provided that copies are not made or distributed for profit or commercial advantage and that copies bear this notice and the full citation on the first page. Copyrights for third-party components of this work must be honored. For all other uses, contact the owner/author(s).

(c) 2021 Copyright held by the owner/author(s).

1539-9087/2021/05-ART36

https://doi.org/10.1145/3448128 


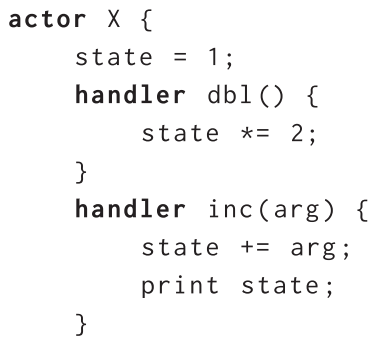

Fig. 1. Pseudo code for an actor network that is deterministic under reasonable assumptions about message passing.

although many are called by other names, including dataflow, process networks, synchronousreactive languages, and discrete-event languages. A narrower definition, originally developed by Hewitt and Agha [1,27], appears in several popular software frameworks such as Scala actors [25], Akka [59], and Ray [52], and programming languages, such as Erlang [4] and P [20]. Unlike various related dataflow models, the Hewitt actor model is nondeterministic. Even if each actor reacts in deterministic ways to incoming messages, when composed, a collection of actors typically yields many possible behaviors. In this article, we explain how the nondeterminism endemic to actors can be avoided without losing expressivity. This results in concurrent (and possibly distributed) programs with more testable and repeatable behaviors that admit nondeterminism only where it is explicitly required by the application.

We begin by illustrating the concern with a simple example, given in Figure 1. It uses a pseudocode syntax that is a mashup of several of the concrete languages mentioned above. This code defines an actor class $X$ that has a single integer state variable that is initialized to 1 . It has two message handlers, named $\mathrm{dbl}()$ and inc(). When invoked, these handlers will double and increment the state variable, respectively. Note that this rather trivial example is purely transformational, but if you replace the number 2 on line 4 with data derived from a sensor and the print state statement on line 8 with something that drives an actuator, then the example becomes reactive. Moreover, a more interesting program would not just send two messages, but rather enter an infinite loop in main where it repeatedly reacts to external stimuli by sending messages. But even this trivial example is sufficient to illustrate the critical problems that arise from the actor model.

The actor named $Y$ with handler main creates an instance of $X$ and sends it two messages, $d b l$ and inc. Note that although many actor languages make these look like remote procedure calls, presumably because such syntax is familiar to programmers, they are not remote procedure calls. Lines 14 and 15 send messages and return immediately, giving a "send and forget" semantics, a key feature that enables parallel and distributed execution.

The program in Figure 1 is deterministic under mild assumptions about the network that relays messages. First, we need to assume that messages are delivered reliably in the same order that they are sent. ${ }^{1}$ Since $\mathrm{dbl}$ is sent before inc, actor $\mathrm{x}$ will execute handler $\mathrm{dbl}($ ) before handler inc(). Second, we need to assume that handlers are mutually exclusive. ${ }^{2}$ That is, once a handler begins executing, it executes to completion before any other handler in the same actor begins executing.

\footnotetext{
${ }^{1}$ This can be realized on a distributed system by relying on the eventual, in-order delivery property of TCP/IP.

${ }^{2}$ This assumption can be relaxed by statically analyzing the code of the handlers and enforcing mutual exclusion only between handlers that share state variables.
} 


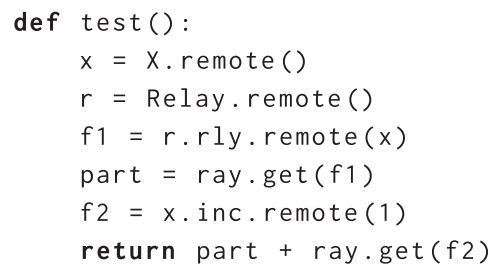

Fig. 4. Alternative test makes program in Figure 3 deterministic.

available in any other actor language that we know of, for controlling the execution of a network of actors. This mechanism could be used, for example, to make the program in Figure 3 deterministic. The test function could be replaced with the code in Figure 4. This code forces the main actor to block until the result of the invocation of $\mathrm{dbl}()$ is received before sending the inc message. This solution, however, requires a very savvy programmer and largely defeats the purpose of the futures. We doubt that many Ray programs will be written with such controls.

This type of nondeterminism is endemic to the Hewitt actor model. Moreover, without the blocking futures of Ray, it is difficult to change the program in Figure 2 to consistently print 3. One way would be to modify class $X$ so it always invokes $\mathrm{dbl}($ ) before inc(), but this is a much more restrictive actor that may as well have only one message handler that doubles the state and then increments it. Alternatively, we could set up another message handler in X that tells it which handler to invoke first, but we would have to ensure that messages to that handler are invoked before any other. Moreover, the semantics now becomes complex. Should a message telling $\mathrm{X}$ to invoke $\mathrm{dbl}$ () first apply only to the next $\mathrm{dbl}$ message or to all subsequent ones? What if two $\mathrm{dbl}$ messages arrive with no intervening inc message?

Since such a simple program results in unfixable nondeterminism, we can only conclude that the Hewitt actor model should be used only in applications where determinism is not required. While there are many such applications, even for those, we pay a price. The code becomes much more difficult to test. Standard testing techniques are based on presenting input test vectors and checking the behavior of the program against results known to be good; in the face of nondeterminism, the entire set of known-good results may be difficult to determine and too vast to enumerate.

To underscore the challenges that nondeterministic software poses to testability, we cite Toyota's unintended acceleration case. In the early 2000s, there were a number of serious car accidents involving Toyota vehicles that appeared to suffer from unintended acceleration. The US Department of Transportation contracted NASA to study Toyota software to determine whether software was capable of causing unintended acceleration. The NASA study [54] was unable to find a "smoking gun," but they concluded that the software was "untestable" and that it was impossible to rule out the possibility of unintended acceleration [31]. The software used a style of design that tolerates a seemingly innocuous form of nondeterminism. Specifically, many state variables, representing for example the most recent readings from a sensor, were accessed unguarded by a multiplicity of threads. We suspect that this style of design seemed reasonable to the software engineers, because one should always use the "most recent" value of a sensor. But the software becomes untestable because, given any fixed set of inputs, the number of possible behaviors is vast.

Not all concurrent software is used in such safety-critical scenarios, of course, but all software benefits from testability. The Toyota software did not use Hewitt actors, but many Hewitt actor programs share a similar form of nondeterminism. Messages are handled in order of arrival, so the state of an actor represents the effects of the "most recent" messages. 
The main contribution of this article is to show that the Hewitt actor model can be extended to yield a deterministic model of computation using any of various techniques, some of which have a long history. These include various dataflow dialects, process networks, synchronous-reactive models, and discrete-event models. After discussing these coordination approaches, we explain a new coordination model we call "reactors," which combines several of the aforementioned techniques with the goal of enabling determinism while preserving much of the style of actors. Specifically, the reactor model promotes modularity and allows for distributed execution. Reactors use a logical model of time to achieve deterministic execution; we show how their capability of relating logical time to physical time allows for the design of distributed reactor systems that behave deterministically.

\section{ACHIEVING DETERMINISM}

A system is deterministic if, given an initial state and a set of inputs, it has exactly one possible behavior. For this definition to be useful, we have to define "state," "inputs," and "behavior." For example, if we include in our notion of "behavior" the timing of actions, then no computer program in any modern programming language is deterministic. In our discussion above, the actor programs have no inputs, the initial state is state $=1$ in an instance of actor X, and the "behavior" is the result printed. Timing is not part of the model and therefore irrelevant to the definition of determinism.

Determinism is a property of a model, not a property of a physical realization of a system [36]. A Turing machine, for example, provides a deterministic model of computation that does not include timing. The "input" is a sequence of bits, and the "behavior" consists of sequential transformations of that sequence. Any particular physical realization of a Turing machine will have properties that are absent from Turing's model, such as timing, but we could construct a different model that did consider timing part of the "behavior." Such a model would be nondeterministic. The same physical system, therefore, is deterministic or not depending on the model.

\subsection{Determinism for Software}

Whether a software system is deterministic depends on our model of the software. A simple model of a program defines initial state as the starting values of all variables, the inputs as a static bit sequence (a binary number) available all at once at the start of execution, and the output as a bit sequence produced all at once upon termination of the program. This is the classic Church-Turing view of computation.

This classic model, however, has difficulty with many practical software systems. A web server, for example, does not have inputs that can be defined as a binary number available all at once at the start of execution. Nor does it terminate and produce a final output. An alternative model for a web server defines its inputs as a (potentially unbounded) sequence of binary numbers, and the "behavior" as sequence of binary numbers produced as outputs. In this model, whether the web server is deterministic may be an important question.

In a concurrent or distributed software system, however, defining the inputs as a sequence of binary numbers may be problematic. A distributed database, like Google Spanner [17], for example, accepts inputs at a globally distributed collection of data centers. It is impossible to tell whether a query arriving in Dallas arrives before or after a query arriving Seattle. ${ }^{3}$ In Google Spanner, however, when a query comes into a data center, it is assigned a timestamp. The "inputs" to the

\footnotetext{
${ }^{3}$ Fundamentally, it is not only difficult to decide which query arrives first, it is impossible to even define what this means. Under the theory of relativity, the ordering of geographically separated events depends on the observer.
} 


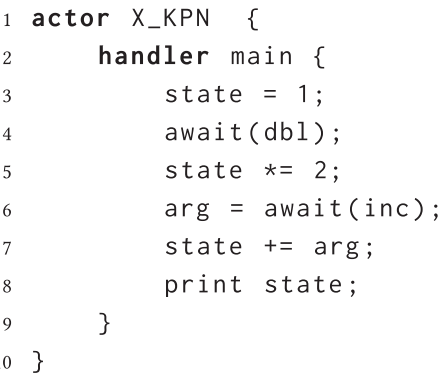

Fig. 5. Variant of $X$ in Figure 2 to encode design intent using blocking reads.

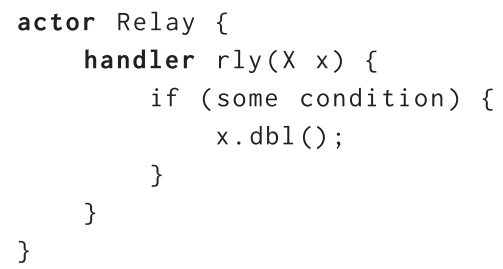

Fig. 6. Modification to actor Relay in Figure 2 to filter messages.

global database are defined as a collection of timestamped queries, and the "behavior" is the set of responses to those queries. Under this model, Spanner is deterministic (under clearly stated assumptions about network behavior). We emphasize that this is not an assertion about any physical realization of Spanner, which could exhibit behaviors that deviate from the model (if, for example, hardware failures undermine the assumptions). It is the model that is deterministic, not the physical realization.

Consider again the actor programs in Figures 2 and 3. If we wish for these programs to be deterministic, then we have to constrain the order in which message handlers are invoked. We have an intuitive expectation that $\mathrm{dbl}()$ should be invoked before inc(), but that is not what the programs say. The programs, as written and as interpreted by modern actor frameworks, do not specify the order in which these handlers should be invoked. Thus, it will not be sufficient to simply improve the implementation of the actor framework. We have to also change the model.

\subsection{Coordination for Determinism}

Let us focus on the actor network sketched in Figure 2. Since actor $Y$ first sends a message that has the eventual effect of doubling the state of actor $X$ and then sends a second message to increment the state of $X$, let us assume that it is the design intent that the doubling occur before the incrementing. Any technique that ensures this ordering across a distributed implementation will require some coordination. There are many ways to accomplish this, many of which date back several decades. Here, we will outline a few of them.

In 1974, Gilles Kahn showed that networks of asynchronously executing processes could achieve deterministic computation; Kahn provided a mathematical model for such processes (Scottcontinuous functions over sequence domains) [29]. In 1977, Kahn and MacQueen showed that a very simple execution policy using blocking reads guarantees such determinacy [30]. Using the Kahn-MacQueen principle, actor X in Figure 2 could be replaced with X_KPN (for Kahn Process Network) in Figure 5. Instead of separate message handlers, a process in a KPN is a single threaded program that performs blocking reads on inputs. The await calls in Figure 5 perform such blocking reads. That code ensures that doubling the state will occur before incrementing it even if actor $Y$ sends its output messages in opposite order.

This way of encoding the design intent, however, has some disadvantages. Suppose that the Relay actor, instead of just relaying messages, filters them according to some condition, as shown in Figure 6. Now the X_KPN will permanently block awaiting a dbl message. The filtering logic would have to be repeated in the X_KPN actor, which would have to surround the blocking read 
of $\mathrm{dbl}$ with a conditional. Moreover, the condition would have to be available now to X_KPN, making the Relay actor rather superfluous. Indeed, our experience building KPN models is that conditionals tend to have to be replicated throughout a network of connected actors, thereby compromising the modularity of the design.

Another family of techniques that is used to coordinate concurrent executions for determinism falls under the heading of "dataflow" and also dates back to the 1970s [19, 53]. Modern versions use carefully crafted notions of "firing rules" [37], which specify preconditions for an actor to react to inputs. Actors can dynamically switch between firing rules governed by some conditions, but once again the conditions need to be shared across a distributed model to maintain coordination. One particularly elegant mechanism for governing such sharing is scenario-aware dataflow, where a state machine governs the coordinated switching between firing rules [65].

Another family of coordination techniques that can deliver deterministic execution uses the synchronous-reactive (SR) principle [6]. Under this principle, actors (conceptually) react simultaneously and instantaneously at each tick of a global (conceptual) clock. Like Kahn networks, the underlying semantics is based on fixed points of monotonic functions on a complete partial order [22] and determinism is assured. Unlike Kahn networks, however, the global clock provides a form of temporal semantics. This proves valuable when designing systems where time is important to the behavior of the system, as is the case with many cyber-physical systems. Some generalizations include multiclock versions [9]. Many projects have demonstrated that despite the semantic model of simultaneous and instantaneous execution, it is possible to implement such models in parallel and on distributed machines using strategies generally called physically asynchronous, logically synchronous (PALS) [64].

A fourth alternative, and the one we focus on in Section 3, is based on discrete-event (DE) systems, which have historically been used for simulation [14, 71], but can also be used as a deterministic execution model for actors. DE is a generalization of SR, where there is a quantitative measure of time elapsing between ticks of the global clock [40] (a concept also found in an extension of Esterel [10]). In fact, Edwards and Hui call this model of a computation a "sparse synchronous" model [21]. In DE models, every message sent between actors has a tag, which is a value drawn from a totally ordered set, and all messages are processed in tag order. The underlying semantics of these models is based on generalized metric spaces rather than complete partial orders, but this semantics similarly guarantees determinism [42].

One challenge when reasoning about DE systems is that we immediately face (at least) two timelines: logical time and physical time(s). Logical time, represented by a tag, is a marker that tracks the processing of events. Physical time is a marker that tracks the movement of physical clocks (not to be confused with the semantic clocks of synchronous-reactive models). The model we discuss in this article assumes that logical time always lags behind physical time; that is, when logical time is advanced, the runtime system waits for physical time to catch up. This aligns the two timelines, but it also comes at a cost. With careful analysis, it is possible to relax this constraint and to allow reacting to events earlier, but this is beyond the scope of this article.

In a distributed system, there are typically multiple physical clocks, which creates a proliferation of time lines. In real-time distributed systems, logical time and physical time can be aligned at selected points (e.g., when taking data from sensors or when actuating some physical devices), but otherwise, logical time is used as a semantic mechanism to ensure deterministic ordering and is allowed to differ from physical time. Unfortunately, natural language makes it difficult to form sentences about more than one time line together. Careful wording is required to avoid confusion, and sometimes, no wording seems adequate, as words like "when" and common expressions such as "at this time" or "at once" become ambiguous. 


\section{REACTORS}

Reactors, introduced by Lohstroh et al. [43-45], are deterministic actors composed of reactions. Reactions bear resemblance to the message handlers used in the previous examples, except rather than responding to messages, reactions are triggered by discrete events and may also produce them. An event relates a value to a tag that represents the logical time at which it is present. Reactions have access to state shared with other reactions, but only within the same reactor. When a reaction executes, it has exclusive access to the reactor's state. Events are the only means by which reactors can communicate with one another, they are always observed in tag order, and events with identical tags are logically simultaneous.

Where message handlers in actors are invoked in no particular order, the order in which reactions can occur is subject to specific constraints. An event can trigger a reaction, but a triggered reaction shall not execute before all observable events with the same tag that might affect it have been produced. This means that any reactions capable of producing such events are forced to execute first (should they be triggered at that logical time). Simultaneously triggered reactions of the same reactor are forced to execute in a predefined order-the order in which they are declared in the reactor's definition. These constraints ensure that reactors react deterministically to inputs, allowing verification of the correctness of their behavior through testing.

\subsection{Lingua Franca}

We are in the process of developing a coordination language called LiNGUA FrANCA (LF), specifically for defining reactors and composing them. As the name suggests, LF is a polyglot "bridge language." In LF, the logic of reactors is given in some target language such as C, Java, or JavaScript. Our LF compiler outputs pure target code and combines it with a runtime implementation (also written in the target language) that coordinates the execution of the program deterministically.

3.1.1 Status and Performance. Although still preliminary, a standalone command-line compiler called $\mathrm{lfc}$ and an Eclipse-based integrated development environment (IDE) for LF are available online. ${ }^{4}$ The compiler is built using the Xtext framework [23]. In addition to generating an Eclipse IDE, Xtext can automatically create editor support for syntax highlighting, content-assist, folding, jump-to-declaration, and reverse-reference lookup across multiple files. It can do this for the Eclipse-based editor, but (some of) these features are also available through a language server, extending the support to any editor that implements the language server protocol. This includes popular ones such as VIM, Emacs, and Visual Studio Code.

To identify the smallest and fastest possible realization of $\mathrm{LF}$, we have developed the $\mathrm{C}$ target, used in all the examples here, despite the fact that $\mathrm{C}$ is a rather low-level language, lacking a strong type system, memory management, and support for object-oriented design. A major goal of developing a $\mathrm{C}$ target is to quantify the minimal cost of supporting the reactor model, a goal for which $\mathrm{C}$ is a suitable choice. An LF program with the $\mathrm{C}$ target is compiled into a standalone $\mathrm{C}$ program that includes the LF runtime system.

The $\mathrm{C}$ runtime consists of about 2,000 lines of extensively commented code and occupies only tens of kilobytes for a minimal application, making it suitable for deeply embedded platforms. We have tested it on Linux, Windows, and Mac operating systems, on both x86-64 and arm64 architectures, as well as on a precision-timed bare-iron platform called Patmos [61]. On platforms that support pthreads (POSIX threads), our multi-threaded runtime environment (approximately 3,000 lines of code) transparently exploits multiple cores while preserving determinism.

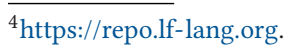


the gray numbered chevrons represent reactions, and the numbers represent the lexical position of the reaction within a reactor (and hence its priority). A white circle represents the startup action, and a triangle represents either a logical or physical action (see Sections 3.5 and 3.6), distinguished by either an "L" or "P." The dashed lines represent dependencies between reactions, and the solid lines represent connections between ports.

\subsection{Ports, Hierarchy, and Actions}

The term "reactors" is a nod to Hewitt actors (actors, revisited), but also to the synchronous reactive programming paradigm that underpins languages such as Esterel [8], SigNAL [7], Lustre [24], and their derivatives. Different from Hewitt actors, reactors do not directly refer to their peers. Reactors have named (and typed) ports that allow them to be connected to other reactors. An event produced by one reactor is only observed by other reactors that are connected to the port on which the event is produced. Events arrive at input ports, and reactions produce events via output ports.

The extra level of indirection implied by ports enables a hierarchical design where a reactor can contain other reactors, such as the one named Composite in Figure 8. The Composite reactor in Figure 8 contains one instance of each of the three other reactors and defines how their ports are connected. In this example, there is only one composite, but composite reactors themselves can also have input and output ports and can be contained by (and connected to) other composites. There is one exception: reactors that are labeled main are not allowed to have inputs or outputs. The main reactor provides the point of entry for executing the LF program. The containment hierarchy of reactors also serves as a scoping mechanism for ports, imposing constraints on the kinds of connections that can be made. Specifically, connections are not allowed to traverse more than one level of hierarchy. For instance, it is possible to connect the input port of a container to the input port of a contained reactor, but not directly to any input ports embedded deeper in containment hierarchy.

Reactors also feature a special variant of ports called actions. Unlike ports, actions are not visible to other reactors and it is not possible to connect to them. Actions are used for scheduling events to trigger reactions of the same reactor at a future logical time. Actions are also used as a synchronization mechanism between reaction code (which executes at well-defined logical time instants) and asynchronous events triggered by the environment, such as interrupts with sensor data, incoming network inputs, or asynchronous callbacks. Such sporadic external events get assigned tags in a way that ensures determinacy, in the sense that once a tag has been assigned, the response of the LF program to the event is well-defined.

In addition to user-defined actions, each reactor has two distinguished triggers: startup, which is present only at the very first time instant of a reactor's execution, and shutdown, which signals the end of a reactor's execution. For example, line 24 in Figure 8 declares a reaction that is to occur at startup.

\subsection{Logical Time}

In the reactor model, each event has a tag. Reactions to events occur at a logical time equal to the tags of the events that are present, and logical time does not advance during a reaction. An input port can have at most one event at any logical time. If during a reaction triggered by an event with some tag $t$ a particular input is absent, then it is guaranteed that no event later appears on that port with a tag equal to (or smaller than) $t$. Any output event produced in a reaction bears the same tag as the input event that triggers the reaction. These outputs, therefore, are logically simultaneous with the triggering inputs, a feature derived from synchronous/reactive models. If any reactor receives more than one event with the same tag (on distinct input ports), then those 


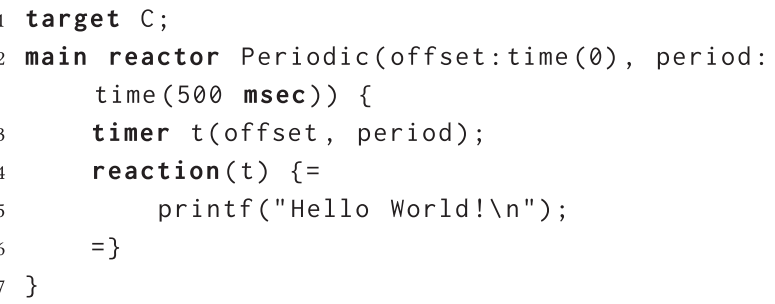

Fig. 9. A reactor that periodically prints "Hello World!".

events are logically simultaneous. If they trigger multiple reactions, then those reactions will be deterministically invoked in the order that the reactions are defined. For example, the reaction on line 24 of Figure 8, which is triggered once at the logical start time of execution, produces two events tagged with the same timestamp. Reactions to those events will be invoked at the same logical time.

The actual representation of time depends on the target language and the execution platform. The only constraint imposed by LF is that time be order-isomorphic with a subset of the natural numbers. This ensures a well-defined notion of simultaneity. In the $\mathrm{C}$ target on POSIX-compliant platforms, time is a 64-bit number representing the number of nanoseconds that have elapsed since January 1, 1970. A tag has, in addition to the time, a microstep, which provides a variant of a superdense model of time $[15,46]$. Each tag is represented by an ordered pair $(t, n)$, where $t$ is a time instant, and $n$ the microstep index. An event with tag $(t, n+1)$ is not logically simultaneous with an event with tag $(t, n)$.

It should be stressed that logical time, in principle, has no relation to physical time, i.e., the passage of time on a wall clock or time measuring device. The reactor model, however, establishes a relationship between logical and physical time at well-defined synchronization points-at the reactor's creation (start) and when interaction with physical processes occurs (see Section 3.6). The first advantage is that it allows LF programs to serve as a specification for time-sensitive tasks, enforce deadlines, and handle deadline misses (see Section 3.7 and 3.8). The second advantage is that the passage of physical time can be used to implement a distributed coordination scheme based on Ptides [73] that, under the assumption of bounded network latency and clock synchronization error, guarantees determinism even across reactors executing on different machines (see Section 5). While the former capability has wide applicability in the domain of embedded software, the latter provides a practical means for improving the robustness and testability of concurrent and distributed systems. Since the advent of the Internet of Things and due to the growth of time-sensitive cloud-based applications, these two worlds have been growing closer to one another, making it particularly attractive to have a programming model like that of LF that suits both.

\subsection{Timers}

In LF, a reactor can have one or more timers, as shown on line 3 of Figure 9. That timer, named $t$, triggers once with a delay equal to the parameter offset (if it is zero, then this will happen at the logical start time of execution), and then once every period. LF has a built-in type for specifying time intervals. A time interval consists of an integer numerical value accompanied with a time unit (e.g., msec for milliseconds or usec for microseconds). Timers are a convenient means for specifying periodic tasks, which are very common in embedded computing. For this reason, timers are offered as a language primitive in LF. In our diagrams, timers are depicted using a clock symbol (e.g., see the Source reactor in Figures 13 and 14). 


\subsection{Logical Actions}

To achieve irregular (not periodic) events where the tag is under program control, an LF program can use a logical action. As mentioned earlier, actions are much like ports, except they cannot be used as endpoints of connections. The events associated with actions originate from the reactor itself, and they appear in response to invocations of a runtime function called schedule. Those invocations can happen either during a reaction or in some asynchronous code. When an event is scheduled to occur for a given action, the value of the tag of the event depends on whether the action is labeled logical or physical.

Logical actions are only intended to be scheduled synchronously, during reactions, as the tag of the resulting event is computed as $t^{\prime}=t+d_{\min }+d$, where $t$ is the current logical time when schedule is called, $d_{\min }$ is the minimum delay specified in the declaration of the action, and $d$ is an additional delay argument provided to the schedule function. This combination of a minimum delay, which is static and statically analyzable, and an additional delay, which can be computed at runtime, offers a balance that enables writing programs with strong guarantees that require static analysis, but also enables programs that require more flexibility.

Logical actions in LF can have an offset of zero. However, this does not result in an event at the same logical time that schedule is called, because this could lead to nondeterminism. Instead, the event will be scheduled at the next microstep. The scheduling of a logical action with zero delay while reacting to an event with tag $(t, n)$, therefore, results in an event with tag $(t, n+1)$.

\subsection{Physical Actions}

Not all invocations of schedule have to happen inside of reaction code that executes at welldefined logical time instants. A reaction can, for instance, pass a callback to a process that carries out some asynchronous activity such as issuing an HTTP request to a remote server or invoking an image classifier that runs on a GPU. In the callback-invoked when a response has been receiveda call to schedule will produce an event, allowing reactions to be triggered by it. An invocation of schedule could also happen directly in an interrupt service routine (ISR), to turn sensor readings into action events, for example.

If an action is labeled physical, then the tags assigned to its events are assigned as follows: Because there is no current logical time, the scheduling will occur relative to a measurement of physical time $T$, I.e., $t^{\prime}=T+d_{\text {min }}+d$. It is probably most common for physical actions to be scheduled without delay, but delays can nonetheless be useful for specifying an amount of physical time to elapse between sensing and actuation.

Note that physical actions make it possible to inject into an executing program tagged events that result from asynchronous physical events outside the program. This goes considerably further than, for example, the timed extension of Esterel by Bourke and Sowmya [10], which provides mechanisms for controlling the timing of the execution of the program and hence for controlling the timing of its effects on the physical world. Physical actions enable more reactive programs; the program can react in predictable ways to external events. As we will explain below in Section 6, this may seem to undermine the determinacy of the LF, but if one considers the tag assigned to these external events as part of the input to the program, then the program remains deterministic. This is the key property that enhances testability; test vectors that include the timing of external events yield exactly one correct response.

The use of physical actions and the distinction between physical and logical actions is sufficiently subtle that we feel compelled to offer an example illustrating the use of both. The example in Figure 10, which can be found in the examples directory on the LF code repository, implements a "reflex game," where a user is presented with a prompt at a random time and asked to respond 


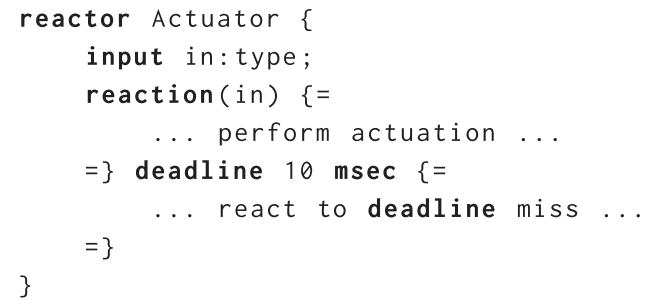

Fig. 12. Schema for a deadline.

will schedule another instance of the prompt action (line 14). Using a logical action in this reactor makes sense, because the reactor itself, not its physical environment, controls the timing of events.

The second reactor, GetUser Input, uses the pthreads library to start a thread that listens for keyboard inputs. The thread is created at startup (line 31). The new thread will, upon detecting that the user has typed Return, schedule the physical action rspns (line 22). That action will be assigned a tag based on the current physical time as reported by the operating system or other time service on the execution platform. The reaction to rspns (line 33) checks to see whether the user cheated and, if not, reports the response time. It then issues a request for another prompt (line 43).

Using a physical action for the second reactor makes sense, because the timing of the events of this action are determined by the physical environment, not by the reactor itself. LF ensures that the tags assigned to these events will not appear "in the past." In other words, it ensures that all reactors see events in timestamp order. The precision with which these logical timestamps match physical time, of course, will depend on the properties of the real-time clock on the execution platform.

\subsection{Deadlines}

The GetUserInput reactor in Figure 10, which turns keystrokes into tagged events through a physical action, is an example of a reactor that wraps a sensor. A typical use case for such a component would be to integrate it into a control system such that it triggers some computation, the result of which ultimately drives an actuator. In Figure 10, the "actuator" just prints to stdout. (line 40). Such control systems are prevalent in automotive applications, fly-by-wire systems in aircraft, and really any kind of cyber-physical system. What these applications typically have in common is that they are subject to a specification that imposes bounds on the maximum latency between sensing and actuation. In an automotive brake system, for instance, the physical time that elapses between the moment of pressing the brake pedal and the brakes being applied has to be bounded to guarantee a braking distance that is considered safe.

In LF, we call these bounds deadlines. A deadline can be specified using the syntax shown in Figure 12. A deadline $D$ (10 msec in the figure) specifies a time interval such that the reaction to the input in with tag $t$ is required to be invoked before physical time $t+D$, as measured on the local platform. In other words, before invoking the reaction to input in at a logical time $t$, the LF runtime system checks the local physical time $T$; if $T \leq t+D$, then it invokes the reaction as usual (line 4). Otherwise, it invokes the code at line 6 that handles a deadline miss. That code could, for example, raise an alarm and/or change the system to operate in a safe degraded mode.

A deadline in an LF program has two roles. First, it provides a hint to the scheduler. Our multicore scheduler for the $\mathrm{C}$ target implements an earliest-deadline-first (EDF) scheduling strategy [13], where every reaction inherits the earliest deadline associated with any reaction downstream of it. 
Second, it provides a mechanism for fault handling, a section of code to invoke if and when the deadline is violated.

If the tag $t$ for the event presented to input in was ultimately derived from a physical action, then the deadline in Figure 12 specifies an end-to-end deadline between sensing and actuation. The deadline may be violated, for example, by excessive execution times of reactions in the path to in, or by poor scheduling decisions that failed to take into account the deadline. Providing assurance that deadlines are not violated requires estimates of worst-case execution time (WCET) of code fragments. LF's architecture naturally breaks down code into fragments, the reactions, which may prove more amenable to WCET analysis than arbitrary programs. We have not implemented such analysis at the time of this writing, however.

\subsection{Scheduling}

Reactions may share state with other reactions in the same reactor. To preserve determinacy, reactions within one reactor are invoked in a predefined order when multiple reactions are triggered logically simultaneously, i.e., by events with identical tags. In LF, this predefined order is the order in which reactions are defined within a reactor definition. This approach follows the sequential constructiveness principle of SCCharts [67] (an extension of Statecharts [26]) which permits sequential reads or writes of shared variables during a synchronous-reactive tick. This principle extends to shared-memory models [2].

Reactions may observe events on ports, actions, or timers that they declare as dependencies, and they may produce events on ports or schedule actions they list as antidependencies. The connections between ports relay events from one reactor to another. For instance, in Figure 8, the Relay reactor's reaction to input $r$ declares that it produces output out using the syntax on line 17 . This means that there is a direct feedthrough relationship from dependency $r$ to antidependency out. Line 17, which is written in the target language $C$ and not examined by the LF compiler, sets the value of the output out equal to the value of the input $r$ at the same logical time as the input event on $r$ that triggers the reaction.

On line 35, port $r$. out is connected to $x . d b l$, meaning there is also a direct feedthrough relationship from $r . r$ to $x . d b l$. More importantly, it means that the first reaction of $X$ is dependent on the reaction of Relay. At the level of the composite, this information can be used to analyze dependencies within the composite and impose scheduling constraints (an algebra for such analysis is given by Zhou and Lee [74]). The purpose of these scheduling constraints is to ensure the deterministic DE semantics even in the presence of parallel and distributed execution.

We impose two constraints on the order of execution of reactors. First, at any logical time where two reactions of the same reactor are triggered, the two reactions must be invoked in the order in which they are defined (and the first reaction must complete before the second reaction starts). Second, if a reaction $r_{1}$ of one reactor declares that it may produce an event that triggers a reaction $r_{2}$ of another reactor, then at any logical time $t$ at which $r_{1}$ is triggered, it shall execute (and finish) before $r_{2}$ starts executing. As long as these two constraints are satisfied, any scheduling policy will preserve determinism. Note that the second constraint is conservative. A reaction $r_{1}$ must declare that it effects an output port $p_{1}$ even if it only sometimes produces an event via $p_{1}$. If, at runtime, it does not actually produce an event at some logical time, then a downstream reaction $r_{2}$, if it is triggered by some other event, must nevertheless wait for the completion of $r_{1}$ before executing. This conservative policy prevents certain scheduling decisions that would have preserved determinacy, so there is a cost. However, there are important benefits that, in our opinion, more than offset these costs.

The most important benefit is that scheduling policies can be developed independently of the target language, enabling the truly polyglot nature of LF. One of the goals of LF is to abstract code 
written in the target language. The code in between $\{=\cdots=$ \} delimiters is not even parsed, much less analyzed by the LF compiler. In principle, it may be possible to infer the declared input/output dependencies from that code if it were to be parsed. However, if the reading of inputs or writing of outputs in a reaction is data dependent, then whether a declared dependency is actually a real dependency proves undecidable. Hence, even the most sophisticated analysis will necessarily be conservative. Our conservative approach, which treats blocks of code in the target language as black boxes, enables support of a variety of target languages using the same model. For example, using $\mathrm{C}$ as a target language is appropriate for resource-constrained, deeply embedded systems, while Python may be a better choice for AI applications, Java for enterprise-scale distributed applications, and JavaScript for Internet of Things applications. Because target-language code is not analyzed in the LF compiler, comparatively little effort is required to add support for new target languages. And despite the code not being analyzed, missing declarations of ports that are referenced inside of reaction code yields errors in the target-language compiler or interpreter, guaranteeing that the declared dependencies are indeed conservative.

Of particular interest are real-time scheduling policies like earliest-deadline-first (EDF) [13] and parallel scheduling on multicore (see Section 4). As discussed in Section 3.7, LF provides a syntax for assigning deadlines to reactions. These deadlines can be used to guide the scheduler. The scheduler in our $\mathrm{C}$ runtime implements a non-preemptive version of EDF by giving precedence to any reaction that is in the critical path of the reaction with the earliest deadline.

\section{PARALLELISM}

The constraints discussed in Section 3.8 can be expressed in a directed acyclic graph, in which the nodes are reactions, and the edges are dependencies between them. This dependency graph ${ }^{7}$ determines, for each reaction, at any given logical time, which reactions (in case they are triggered) have to precede it. Because the dependency graph establishes a partial order of reactions, it may be allowable to execute certain reactions in parallel. Specifically, if two distinct reactors receive logically simultaneous events, then their reactions may be invoked in parallel unless there is a direct dependency between them.

The reactor runtime environment for LF automatically exploits parallelism exposed by the (lack of) dependencies between reactions in the dependency graph. The runtime system has two priority queues: an event queue and a reaction queue. Events are scheduled by inserting them into the event queue, in which events are ordered by tag. Events are bound to timers or actions (recall, actions are like ports, but only visible internally) and can trigger reactions. Reactions, in turn, can use actions to schedule events at a future logical time instant (which get loaded onto the event queue). A value written to a port produces an instantaneous event that could trigger dependent reactions in downstream reactors. Triggered reactions are inserted in the reaction queue and are ordered based on their position in the dependency graph. The runtime system maintains a thread pool with workers and maps reactions that it pops from its reaction queue onto workers that execute them. Figure 13 shows a typical scatter/gather pattern, where all of the Compute reactors can be executed in parallel provided there is a sufficient number of workers to do so. A simple strategy is to match the number of workers to the number of available cores.

In Figure 14, a chain of reactions is triggered by a timer with a specified period. Each reaction produces an output event that is logically simultaneous with its input, but each stage of the

\footnotetext{
${ }^{7}$ In a dependency graph, a directed edge denotes a "depends on" relationship between two nodes. A dependency graph can also be encoded as a precedence graph, in which directed edges denote a "happens before" relationship. These representations are toplogically identical, but the polarity of the edges is inverse. In the context of concurrency control in databases, precedence graphs are also commonly referred to as "conflict graphs" or "serializability graphs."
} 


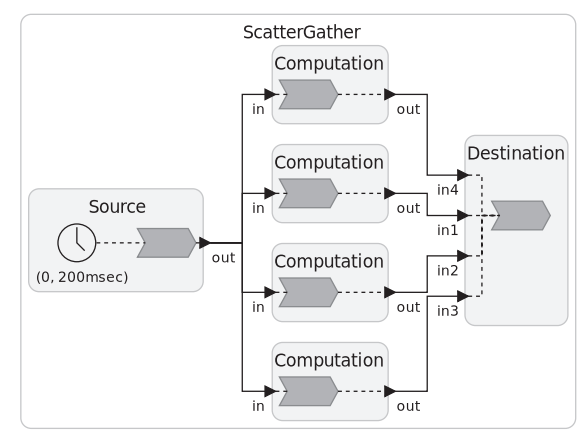

Fig. 13. An LF program realizing a scatter/gather pattern.

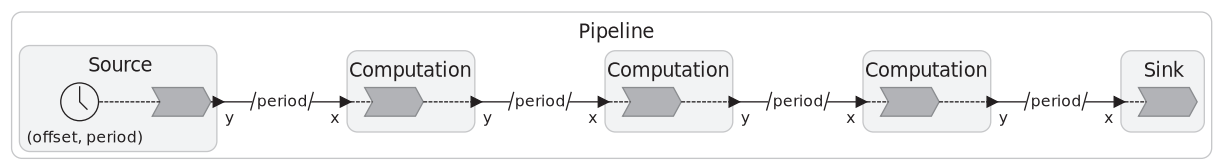

Fig. 14. An LF program that is easy to execute in parallel using pipelining.

pipeline is broken up by a delay specified by the parameter period. This effectively breaks up the dependency graph into disconnected subgraphs. At each tag, there is no dependency between any two reactions in the pipeline, so they can be executed in parallel.

\section{DISTRIBUTED EXECUTION}

Discrete-event models of computation, where timestamped events are processed in timestamp order, have been used for simulation for a long time $[14,71]$. There is also a long history of executing such simulations on parallel and distributed platforms, where the primary challenge is maintaining the timestamp ordering without a centralized event queue. The classic Chandy and Misra approach [16] assumes reliable eventual in-order delivery of messages along any path from an output port to an input port. It requires each actor with two or more input ports to defer processing any timestamped input message until each of its inputs has at least one pending input message. It is then safe to process the message with the least timestamp. To avoid starvation, the Chandy and Misra approach requires that "null messages" be sent periodically on every channel so no actor is blocked indefinitely waiting for messages that will never arrive.

The Chandy and Misra approach is the centerpiece of a family of so-called "conservative" distributed simulation techniques. An alternative, first described by Jefferson [28], is to use speculative execution. Jefferson's so-called "time warp" approach relies on checkpointing the state of all actors and the event queue and then handling timestamped messages as they become available. As messages are handled, the local notion of "current time" is updated to match the timestamp of the message. If a message later becomes available that has a timestamp earlier than current time, then the simulation is rolled back to a suitable checkpoint and redone from that point.

A third approach, the High-Level Architecture (HLA) [32], uses a centralized broker to regulate the advancement of logical time in each "federate" of a "federation." Each federate queries the broker when it wishes to advance its local notion of logical time, and it exposes to the broker information about future events it may produce.

While all of these techniques are effective for simulation, they have serious disadvantages for reactors, which are intended to be used as system implementations, not as simulations. In addition to the overhead of null messages, the Chandy and Misra approach suffers the more serious 


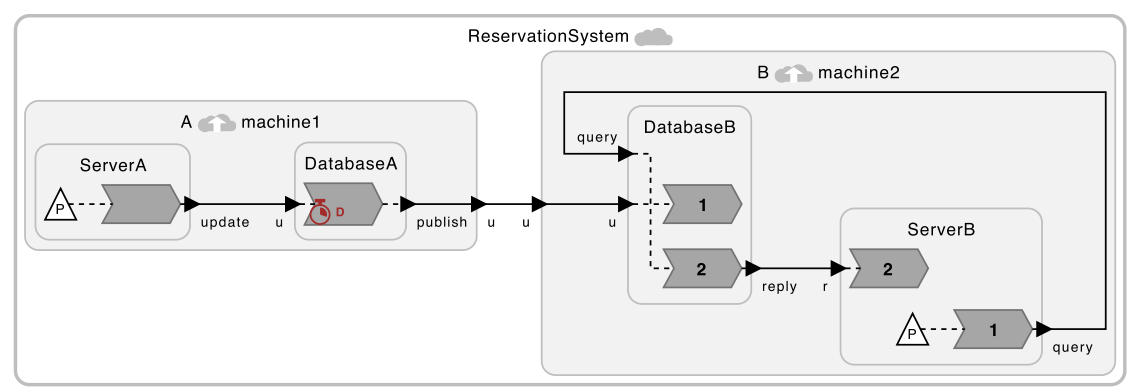

Fig. 15. Distributed reactors implementing a reservation system using Ptides.

disadvantage that every node in a distributed system becomes a single point of failure. If any node stops sending messages, then all other nodes will eventually grind to a halt, unable to proceed while they wait for null or real messages. In addition to the overhead of redoing execution, the time warp approach suffers the more serious disadvantage that in a system deployment, unlike a simulation, some actions cannot be rolled back, particularly those with side effects in the physical world. In the HLA approach, like Chandy and Misra, every federate is a single point of failure. Also, the broker (often called the "runtime infrastructure" or "RTI") can become a performance bottleneck.

To address these concerns, Zhao et al. introduced Ptides, a programming model for distributed real-time system realizations (not simulations) that is compatible with reactors [73]. Ptides was later independently reinvented at Google, where it became the backbone of a globally distributed database system called Spanner [17].

Ptides and Spanner make two key assumptions about the execution platform. First, they assume that each node in the distributed system has a physical clock that is synchronized with that of all other nodes, and that there is a bound $E$ on the clock synchronization error. That is, if you simultaneously ask two nodes what time it is, they will not disagree by more than $E$. Second, they assume that every network connection between nodes has a bound $L$ on the latency for message delivery. This assumption is necessary anyway for many realtime applications.

These two assumptions, $E$ and $L$, may, of course, be violated in any physical deployment of a physical system. Hardware failures or malicious attacks, for example, could cause violations. One interesting property of reactors is that such violations are detectable. They result in out-of-order timestamps. This condition can be detected at runtime as a fault condition, enabling fault-tolerant system designs that adjust themselves to such fault conditions. Moreover, the assumptions $E$ and $L$ are explicit and quantified. Many practical system designs make such assumptions implicitly and without quantification, making detection of violations difficult.

We can use Spanner's database application in a simple scenario depicted in Figure 15 to explain how these two assumptions enable efficient and deterministic distributed execution. Consider a distributed database, e.g., a reservation system, where the data is replicated on two different machines. The first machine hosts a Lingua Franca program A and the second machine hosts another program B. A and B each contain a copy of the database and a web server. Assume that A's server accepts updates to the database, for example to reserve an airline seat. Assume that B's server accepts queries for records in the database, for example to determine whether a particular seat is available. Assume that the two copies of the database are initially identical.

Suppose an update to a record arrives through ServerA and that, around the same time, a query arrives through ServerB for the same record. In Ptides and Spanner, these two events will be assigned tags based on the physical clocks on the two machines, and the correct response from the database will depend on the numerical order of these two tags. 
In the figure, both servers are shown to be triggered by a physical action (denoted by the triangle with a "P" in it). When one of these servers receives an input from the outside world, it calls the schedule function to schedule an event for its physical action. That event triggers a reaction in the server that produces either an update or query output. The tag $t$ of the event (and hence also of the output) is obtained from the local physical clock. In A, the update output goes to the database, which updates its local copy of the record and sends a publish output to $B$ to notify the other copy of the database of the update. In B, the query output from the server goes to the query input of the database, which reacts by sending a reply back to the server containing the value of the queried record.

The key question is this: If these two events occur nearby in time, how can we ensure that if the tag of the update is less than or equal to the tag of the query, so the reply will be the updated record value, not the old value?

The publish message is sent from machine 1 to machine 2 , so it incurs network latency that is assumed to not exceed some number $L$. Furthermore, the reaction in DatabaseA has a deadline $D$, indicated by the small red clock symbol in the reaction. Assuming that the execution time of the reaction in Database $\mathrm{A}$ is included in $L$, then the event will arrive at machine 2 before physical time on machine 1 exceeds $t_{u}+D+L$, where $t_{u}$ is the tag of the update event. Because the clock synchronization error between the machines is bounded by $\mathrm{E}$, the time indicated by the physical clock of machine 2 upon arrival of the update message from machine 1 will not exceed $t_{u}+D+L+E$.

As a consequence, when machine 2's physical clock hits $t_{u}+D+L+E$, it can assume it has received all database updates with tags less than or equal to $t_{u}$. At that physical time, it is "safe to process" any event on machine 2 with tag less than or equal to $t_{u}$.

Suppose that, around the same time, ServerB receives a query for a record from the outside world and, using the same physical action mechanism, assigns it tag $t_{q}$. DatabaseB, however, cannot safely reply to that query until the physical clock at machine 2 matches or exceeds $t_{q}+D+L+E$. Hence, before acting on that query, machine 2 simply waits until its physical clock hits that threshold. Then it invokes reaction 1 of ServerB, which sends that query to DatabaseB, which invokes its reaction 2 to produce a reply, which then in turn invokes reaction 2 of ServerB to send a reply to the outside world. If $t_{u} \leq t_{q}$, then the correct reply is the updated record. Otherwise, the reply is assured of being the value of the record before the update.

Such a distributed system could instead use the Chandy and Misra approach, which would require A to periodically send tagged null messages to B. Then, DatabaseB will repeatedly receive null messages on its update port with steadily increasing tags. As soon as one of those tags exceeds $t_{q}$, it can handle the event on its query port that has tag $t_{q}$ and send a reply back to WebServer. However, as we have pointed out, the Chandy and Misra approach has high overhead and is vulnerable to node failures.

In Ptides and Spanner, the approach instead is to watch the local clock and to hold off processing the query message until its measurement of physical time exceeds the "safe-to-process" threshold $S T P=t_{q}+D+L+E$.

This mechanism is implemented in LF, which also implements a centralized coordination mechanism similar to HLA that does not require clock synchronization. These mechanisms have a number of subtleties that are beyond the scope of this article, particularly when there are feedback loops between federates and when deadlines are involved. But for this simple use case, perhaps the only subtlety of significance concerns what happens when the assumptions are violated. If the network latency, for example, exceeds $L$, then it becomes possible for machine 2 to receive a message with tag $t_{u}$ after it has processed a message with tag $t_{q}<t_{u}$. In LF, this is a fault condition. A fault 
handler can be provided as part of the program to recover from the fault, for example by overlaying a transaction schema on the database and rejecting a transaction.

Note that on machine 2, there is a physical delay of STP $=D+L+E$ before a query will trigger a reply. Suppose that an engineering requirement for the design of this database is that the database should reply to queries within 30 milliseconds. Then, using this Ptides/Spanner design, this translates into an engineering requirement that $D+L+E \leq 30 \mathrm{msec}$. This provides guidance for selection of processing and networking technology and provides a clear criterion for determining what hardware can correctly execute this system with the timing requirements.

If any of the assumptions, $D$ (an execution time bound), $L$ (a network latency bound), or $E$ (a bound on the clock synchronization error) is violated, then a fault may occur. The rarity of the occurrence can be controlled by standard engineering methods. The more aggressive the bounds for the underlying technology, the more likely the faults. But, of course, faults cannot be made impossible. No matter what assumptions you make about the realization of a system, those assumptions may get violated in the field.

Another clear advantage of this approach is that reads to the database generate no network traffic. Only writes that update records generate network traffic.

The work by Menard et al. [51] shows how a strategy very similar to the one described in this article corrects nondeterminism in a real-world application, namely, a brake assistant demonstrator application that is provided by the AUTOSAR consortium. The same solution can be applied to our example in Figure 2. In that example, the messages are all logically simultaneous (they bear the same tag). If the three actors are executed on distributed machines, then when the machine executing $\mathrm{X}$ receives an inc message with tag $t_{m}$, it should not invoke the message handler until the local clock exceeds $t_{m}+\max \left(D_{1}, D_{2}\right)+E+L$, where $D_{1}$ and $D_{2}$ are the deadlines associated with the reactions that send messages. The use of hierarchy ensures that there is a software entity, the container for the three actors, that "knows" the topology, and the use of ports with causality interfaces ensures that the dependency analysis required to derive this threshold can be performed. If bounds on execution times are derivable from the code [69], then $D_{1}$ and $D_{2}$ can also be derived automatically. Or the system could be realized using PRET machines [38], in which case extremely high confidence in the bounds on the execution times becomes achievable.

The cost of determinism in this case is increased latency, because the machine executing $X$ must wait for physical time to pass. If this cost is too high, and nondeterminism is tolerable, then a simpler mechanism can be used that reassigns tags at the receiver using the local physical clock. This will introduce nondeterminism, but only with respect to messages exchanged between reactors mapped to different machines. Interactions between reactors that are executed on the same machine (even a multicore machine) remain deterministic. The principle we advocate is that the system designer should choose to make the system nondeterministic, rather than having this decision forced by the framework. Moreover, once a tag is assigned at the receiving end, the behavior of the system is deterministic. As a consequence, even a nondeterministic design becomes testable, because input test vectors can include the assigned tags as part of the test vector.

\section{SEMANTICS}

LF, with its timestamped events, is rooted in a discrete-event model of computation. We can leverage prior work with the semantics of discrete-event systems [15, 33, 41, 49, 50, 58, 70, 72] to prove determinism. A program is deterministic if it exhibits exactly one behavior for each set of inputs, Some care is needed, however, because this statement requires defining precisely what we mean by "behavior" and "input." 
First, LF cannot be fully dealt with by the DEVS formalism of Zeigler et al. [72], because there is no requirement for a nonzero logical time delay from inputs to outputs of reactors. Outputs are simultaneous (in logical time) with inputs, much like the synchronous languages [6].

Second, LF uses a superdense model of time [46, 48], where there is no requirement for a delta-causal component in feedback loops. As a consequence, the metric-space semantics of References $[33,58,70]$, which uses the Cantor metric and the Banach fixed point theorem, cannot be used unmodified. We can choose a semantics based on complete partial orders (CPOs) [41] or on a generalized ultrametric space $[15,49,50]$. Here, we choose the latter. We will not give the full formalism here, since it is well documented in the literature, but instead will only explain how to map LF onto this formalism. A full understanding will require reading the prior work.

We use the concept of a signal to represent the sequence of timestamped messages that flow from output ports to input ports in LF. Formally, a signal is a partial function $s: T \rightarrow V$, where $T$ is the tag set and $V$ is the set of possible message values. A signal is defined for tags where there is an event (a message is sent) and is undefined for other tags. For the purposes of proving determinism, we take "behavior" to be the set of signals produced by a program execution.

The prior work with ultrametric space semantics assumes a superdense time tag set $T=\mathbb{R} \times \mathbb{N}$, but the theory applies for any totally ordered set. There are no real numbers in LF, so the tag set can be accurately modeled by $T=\mathbb{N} \times \mathbb{N}$, where $\mathbb{N}$ is the set of natural numbers. The set is ordered lexicographically. Dispensing with real numbers means that some of the corner cases that arise in a generalized ultrametric semantics do not arise in LF. One subtlety that we do not escape, however, is the possibility of Zeno systems, where one part of the system fails to advance time past a certain finite point while another part of the system proceeds beyond that point. Consider a program where one portion advances time only by microsteps and another by metric time. It can be shown that whether or not a given program is Zeno is undecidable; a clever demonstration of this has been given by Ben Lickly who gives an example discrete-event program that is Zeno if the Collatz conjecture is false and non-Zeno if it is true [35]. Since Zeno systems are probably not useful, we will simply assume that our semantics does not include Zeno systems. The only thing remaining to do is prove that a program is modeled by a strictly contracting endofunction in the generalized ultrametric space. Determinism will then follow from the existence and uniqueness of a fixed point for this function.

First, we have to show that each reactor is indeed modeled by a function. This function has the form

$$
F:(T \rightarrow V)^{N} \rightarrow(T \rightarrow V)^{M},
$$

where $(T \rightarrow V)$ is the set of all signals, $N$ is the number of input ports, and $M$ is the number of output ports. Some care is needed here, because a reaction contains arbitrary code in a target language, code that LF is not concerned with. If that code is nondeterministic, e.g., by invoking a random number generator seeded by the current time, then it is far from obvious how to model the reactor as such a function. But recognizing that our goal is to show the LF is deterministic (it does not introduce nondeterminism), not that the target language is deterministic, for each execution of the program, we can take the function to be the one determined by the particular outcome of every nondeterministic choice in the target language. This is analogous to the way the prior DE semantic models handle external inputs. For each execution, the function realized by each component is determined, in part, by the particular external inputs provided to that execution. For the example of the random number generator, we can consider the seed to be an external input. The function will be different for each execution of the program because the input will be different, but it will be a function nonetheless. 
A similar strategy can be used to handle physical actions, which get assigned a timestamp based on the current physical clock of the executing platform. The function realized by a reactor will depend on that timestamp, so function will be different for every execution, but it is nevertheless a function, rendering the theory applicable. Hence, the timestamp, not just the value, of a physical action is considered an external input to the program. Given the inputs, including the timestamps assigned to physical actions, the behavior of the program will prove deterministic, an extremely valuable property (consider that it enables regression testing, for example).

A final subtlety is that LF allows reactions to overwrite an output produced by a previous reaction. Since these two output values have the same timestamp, this would seem to make it impossible to model an output signal as a function whose domain is the set of timestamps. However, because of the dependency analysis, which constrains the execution order of reactions, no other reactor will see the first value. Every other reactor sees only the final value at any timestamp, and hence there is no contradiction. That final value is the output from the function $F$ (this also explains why we model reactors, not reactions as functions). We next need to show that for every execution, the $F$ function for each reactor is contracting in a generalized ultrametric space. Following existing approaches in the literature [15, 41,49], we define the generalized ultrametric over the set $(T \rightarrow V)^{N}$ of $N$-tuples of signals to be a function

$$
d:(T-V)^{N} \times(T \rightarrow V)^{N} \rightarrow \Gamma,
$$

where $\Gamma$ is the set of downsets of the tag set $T$, and $N$ is a positive integer. For a particular pair of tuples of signals $\mathbf{s}_{1}, \mathbf{s}_{2}, d\left(\mathbf{s}_{1}, \mathbf{s}_{2}\right)$ is the largest downset of $T$ where the restrictions of $\mathbf{s}_{1}$ and $\mathbf{s}_{2}$ to this downset are equal. In other words, $d\left(\mathbf{s}_{1}, \mathbf{s}_{2}\right)$ is the tag set of the largest common prefix of $\mathbf{s}_{1}$ and $\mathbf{s}_{2}$.

The set $\Gamma$ is totally ordered by reverse set containment. Thus, for $\gamma_{1}, \gamma_{2} \in \Gamma$, we write $\gamma_{1} \leq \gamma_{2}$ if and only if $\gamma_{1} \supseteq \gamma_{2}$. A function $F$ modeling a reactor is a contraction if for all $N$-tuples $\mathbf{s}_{1}, \mathbf{s}_{2} \in(T \rightarrow V)^{N}$,

$$
d\left(F\left(\mathbf{s}_{1}\right), F\left(\mathbf{s}_{2}\right)\right) \leq d\left(\mathbf{s}_{1}, \mathbf{s}_{2}\right) .
$$

In words, the tag set of the common prefix of two possible outputs from the function is at least as big as the tag set of the common prefix of the two possible inputs that produce these outputs. This property is trivially satisfied by all LF reactors, because outputs cannot depend on events with tags larger than that of the output. In other words, every reactor is causal (no output event depends on a future input event, one with a larger tag).

One final step is needed. Using the connections between ports to guide function composition, the individual functions $F_{r}$ for each reactor $r$ can be systematically composed to construct a function

$$
G:(T \rightarrow V)^{P} \rightarrow(T \rightarrow V)^{P},
$$

where $P$ is the total number of signals in the program and $G$ describes the entire program. The procedure for constructing this function $G$ is systematic (see Lee and Seshia [39], Chapter 6).

An example fashioned after Figure 6.1 of Lee and Seshia [39] is given in Figure 16. Figure 16(a) shows a cyclic composition of four reactors producing four signals $(P=4)$. Each reactor is modeled by a function $F_{1}$ through $F_{4}$. These functions are assembled in parallel in Figure 16(b) to define an endofunction $G$ that has the four signals as inputs and outputs. Four feedback connections then route each output to the corresponding input. The constraints of LF ensure that the graph of reactions (not reactors) is acyclic (any feedback loop in the dependency graph between reactions must include at least one microstep delay), and hence there always exists a finite unrolling (the function $G$ applied to its own outputs some number $N$ times) such that there is no path through the resulting graph of reactions from any input to the first $G$ to any output of the last $G$. Since there is no such path, at each logical time, each output from $G^{N}$ at each logical tag does not depend on 


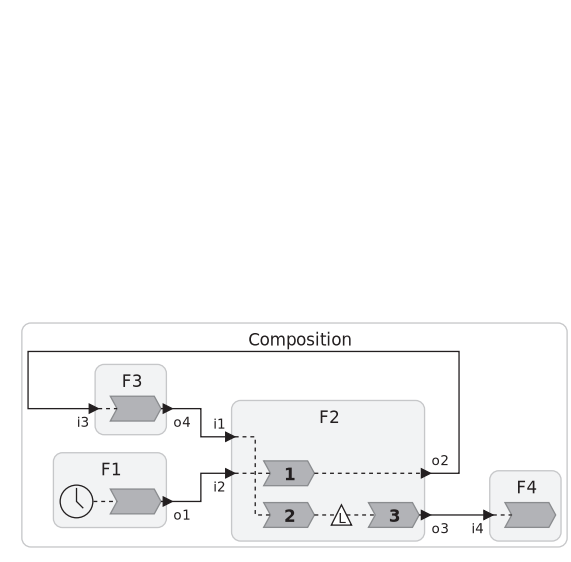

(a) Normal composition

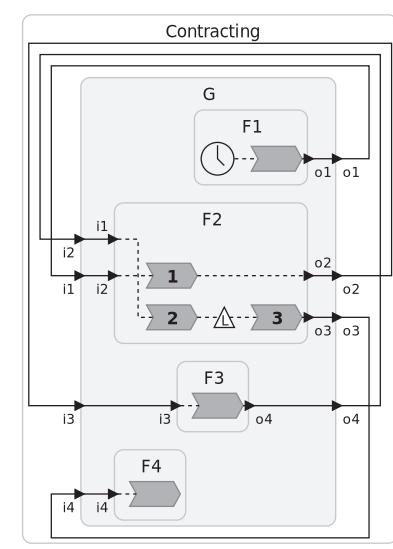

(b) Feedback composition

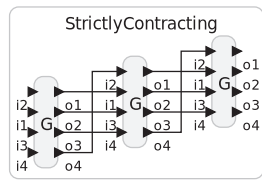

(c) Unrolled feedback

Fig. 16. Example of the construction of a strictly contracting function $G^{N}$ modeling an LF program.

any input at that logical tag. In the example, $N=3$ is sufficient. In general, it is easy to show that $N$ is no larger than the total number of reactions in the program.

Since any parallel composition of contracting functions is contracting, $G$ is a contracting function. The function $G^{N}$, however, is strictly contracting because of the lack of direct paths from any input to any output. Hence, every LF program can be modeled as a feedback loop with a strictly contracting function $G^{N}$ mapping all signals to all signals. A classic fixed point theorem [55] tells us that such a function has exactly one fixed point, and hence there can be only one set of signals that satisfy the program. Hence, the program is deterministic. That fixed point theorem, however, is not constructive (it gives no way to find the fixed point). In Cataldo et al. [15], the classic Banach fixed point theorem, which applies to ordinary metric spaces, is generalized to apply to generalized ultrametric spaces. That theorem is constructive. The constructive procedure for finding the fixed point offers an operational semantics for LF, while the existence and uniqueness of the fixed point gives a denotational semantics. These two semantics match and hence are "fully abstract."

For completeness, one final observation is in order. LF permits the structure of programs to change at runtime through a mechanism called mutations $[43,44]$. Logically, these mutations can be modeled as occurring between logical timesteps because, at each timestep, the mutations always precede any reaction that may be affected by the mutation. Semantically, this is termination of the execution of one deterministic program at the conclusion of a logical timestep and starting a new deterministic program at the next logical timestep. Two or more distinct functions $G$ participate in determining the behavior of the program. As long as the timestep is chosen deterministically and the mutation itself is a function of the inputs, the result is still a deterministic program.

\section{RELATED WORK}

The use of synchronous-reactive principles to deterministically coordinate concurrent software has a long history, with notable contributions such as Reactive C [11], SL [12], SyncCharts [3], and ReactiveML [47]. A modern variant of SyncCharts, SCCharts [66], composes finite state machines under a synchronous semantics. It has been recently augmented with a semantic notion of time [62] based on the concept of dynamic ticks [68]. Like reactors, components can inform the scheduler at what logical time to trigger reactions.

Also related is a family of so-called "active object" languages [18], which approach the problem of concurrent execution by generalizing object-oriented programming with asynchronous method 
calls and (sometimes) futures, techniques that allow for parallel and distributed execution. Ensuring determinacy, however, is not a priority, and even support for avoiding the common pitfalls of threads [34] is sparse in some of these languages.

The concept of "reactive isolates" [57] (later also called "reactors") was introduced to modularly combine different communication protocols inside the same actor (realized in the Scala-based Reactors.IO framework [56]). A key difference with Hewitt actors is that reactive isolates have separate channels for receiving messages from other actors and internal event streams to compose reactions. Their channels are analogous to our input ports. They have no analogy to our output ports, however. A channel in reactive isolates is a direct reference to an isolate that other isolates can send messages to. Like classic actors, reactive isolates do not feature a semantic notion of time, and their communication is asynchronous with no guarantees on message arrival order.

\section{CONCLUSION}

Reactors are a variation on actors that leverage logical time, following classical synchronousreactive principles, to achieve determinism. The reactor model also relates logical time to physical time, which allows for the specification of real-time constraints, as well as the preservation of determinism in distributed programs via the application of safe-to-process analysis known from Spanner and Ptides. While the emphasis of reactors is on determinacy, asynchrony and nondeterminism can be realized, when needed, by scheduling events relative to readings obtained from a physical clock. The assumptions that need to be satisfied to achieve deterministic computation across a distributed platform are explicit in LF. This makes the language well suited to safetycritical applications, because it decouples the problem of getting the program logic correct from the problem of selecting and configuring hardware that meets the assumptions. Moreover, LF programs are testable. Unless assumptions are broken or nondeterministic behavior is specified explicitly, given timestamped input test vectors, LF programs yield deterministic timestamped output vectors.

\section{ACKNOWLEDGMENTS}

The authors would like to acknowledge and thank the following people for their contributions to the design and implementation of Lingua Franca: Alexander Schulz-Rosengarten (Kiel University) who built the interactive diagram synthesis tool in our IDE; Matt Weber (UC Berkeley) who has worked on code generation and has helped develop the TypeScript runtime; Shaokai Lin (UC Berkeley) who is creating a UCLID5 [63] target with the goal of enabling model checking LF programs. Others who have influenced LF with their ideas are: Janette Cardoso, Jeronimo Castrillon, Patricia Derler, Christopher Gill, Andrés Goens, Íñigo Íncer Romeo, Alberto SangiovanniVincentelli, Martin Schoeberl, Sanjit Seshia, and Marjan Sirjani. We also thank the anonymous reviewers for their helpful comments on earlier versions of this manuscript.

\section{REFERENCES}

[1] Gul A. Agha, Ian A. Mason, Scott F. Smith, and Carolyn L. Talcott. 1997. A foundation for actor computation. f. Funct. Prog. 7, 1 (1997), 1-72.

[2] Joaquín Aguado, Michael Mendler, Marc Pouzet, Partha Roop, and Reinhard von Hanxleden. 2018. Deterministic concurrency: A clock-synchronised shared memory approach. In Programming Languages and Systems (ESOP), Vol. LNCS 10801. Springer. DOI : https://doi.org/10.1007/978-3-319-89884-1_4.

[3] Charles André. 1996. SyncCharts: A Visual Representation of Reactive Behaviors. Report RR 95-52. University of SophiaAntipolis.

[4] Joe Armstrong, Robert Virding, Claes Wikström, and Mike Williams. 1996. Concurrent Programming in Erlang (2nd ed.). Prentice Hall.

[5] Henry C. Baker Jr and Carl Hewitt. 1977. The incremental garbage collection of processes. ACM Sigplan Not. 12, 8 (1977), 55-59. 
[6] Albert Benveniste and Gérard Berry. 1991. The synchronous approach to reactive and real-time systems. Proc. IEEE 79, 9 (1991), 1270-1282.

[7] Albert Benveniste and Paul Le Guernic. 1990. Hybrid dynamical systems theory and the SIGNAL language. IEEE Trans. Autom. Contr. 35, 5 (1990), 525-546.

[8] Géard Berry and Georges Gonthier. 1992. The ESTEREL synchronous programming language: Design, semantics, implementation. Sci. Comput. Prog. 19, 2 (Nov. 1992), 87-152. DOI : https://doi.org/10.1016/0167-6423(92)90005-V.

[9] Gerard Berry and Ellen Sentovich. 2001. Multiclock Esterel. In Correct Hardware Design and Verification Methods (CHARME), Vol. LNCS 2144. Springer-Verlag.

[10] Timothy Bourke and A. Sowmya. 2009. Delays in Esterel. In SYNCHRON, Vol. Seminar 09481.

[11] Frédéric Boussinot. 1991. Reactive C: An extension to C to program reactive systems. Softw. Pract. Exper. 21, 4 (Apr. 1991), 401-428.

[12] Frédéric Boussinot and Robert de Simone. 1996. The SL synchronous language. IEEE Trans. Softw. Eng. 22, 4 (Apr. 1996), 256-266.

[13] Giorgio C. Buttazzo. 2005. Hard Real-time Computing Systems: Predictable Scheduling Algorithms and Applications (2nd ed.). Springer.

[14] C. G. Cassandras. 1993. Discrete Event Systems, Modeling and Performance Analysis. Irwin.

[15] Adam Cataldo, Edward A. Lee, Xiaojun Liu, Eleftherios Matsikoudis, and Haiyang Zheng. 2006. A constructive fixedpoint theorem and the feedback semantics of timed systems. In Proceedings of the Workshop on Discrete Event Systems (WODES'06).

[16] K. Mani Chandy and Jayadev Misra. 1979. Distributed simulation: A case study in design and verification of distributed programs. IEEE Trans. Softw. Eng. 5, 5 (1979), 440-452.

[17] James C. Corbett et al. 2013. Spanner: Google's globally-distributed database. ACM Trans. Comput. Syst. 31, 8 (2013).

[18] Frank S. de Boer, Vlad Serbanescu, Reiner Hähnle, Ludovic Henrio, Justine Rochas, Crystal Chang Din, Einar Broch Johnsen, Marjan Sirjani, Ehsan Khamespanah, Kiko Fernandez-Reyes, and Albert Mingkun Yang. 2017. A survey of active object languages. Comput. Surv. 50, 5 (2017), 76:1-76:39.

[19] Jack B. Dennis. 1974. First Version Data Flow Procedure Language. Report MAC TM61. MIT Laboratory for Computer Science.

[20] Ankush Desai, Vivek Gupta, Ethan Jackson, Shaz Qadeer, Sriram Rajamani, and Damien Zufferey. 2013. P: Safe asynchronous event-driven programming. ACM SIGPLAN Not. 48, 6 (2013), 321-332.

[21] Stephen Edwards and John Hui. 2020. The sparse synchronous model. In Proceedings of the Forum for Specification and Design Languages (FDL'20). IEEE, 1-8.

[22] Stephen A. Edwards and Edward A. Lee. 2003. The semantics and execution of a synchronous block-diagram language. Sci. Comput. Prog. 48, 1 (2003), 21-42.

[23] Moritz Eysholdt and Heiko Behrens. 2010. Xtext: Implement your language faster than the quick and dirty way. In Proceedings of the ACM International Conference Companion on Object-oriented Programming Systems Languages and Applications. ACM, 307-309.

[24] N. Halbwachs, P. Caspi, P. Raymond, and D. Pilaud. 1991. The synchronous data flow programming language LUSTRE. Proc. IEEE 79, 9 (1991), 1305-1319.

[25] Philipp Haller and Martin Odersky. 2009. Scala actors: Unifying thread-based and event-based programming. Theoret. Comput. Sci. 410, 2-3 (2009), 202-220.

[26] David Harel. 1987. Statecharts: A visual formalism for complex systems. Sci. Comput. Prog. 8, 3 (1987), $231-274$.

[27] Carl Hewitt. 1977. Viewing control structures as patterns of passing messages. f. Artif. Intell. 8, 3 (1977), 323-363.

[28] D. Jefferson. 1985. Virtual time. ACM Trans. Prog. Lang. Syst. 7, 3 (1985), 404-425.

[29] Gilles Kahn. 1974. The semantics of a simple language for parallel programming. In Proceedings of the IFIP Congress. North-Holland Publishing Co., 471-475.

[30] Gilles Kahn and D. B. MacQueen. 1977. Coroutines and networks of parallel processes. In Information Processing, B. Gilchrist (Ed.). North-Holland Publishing Co., 993-998.

[31] Philip Koopman. 2014. A Case Study of Toyota Unintended Acceleration and Software Safety. Retrieved from http: //betterembsw.blogspot.com/2014/09/a-case-study-of-toyota-unintended.html.

[32] Frederick Kuhl, Richard Weatherly, and Judith Dahmann. 1999. Creating Computer Simulation Systems: An Introduction to the High Level Architecture. Prentice Hall PTR.

[33] Edward A. Lee. 1999. Modeling concurrent real-time processes using discrete events. Ann. Softw. Eng. 7 (1999), 25-45.

[34] Edward A. Lee. 2006. The problem with threads. Computer 39, 5 (2006), 33-42.

[35] Edward A. Lee. 2014. EECS 219D: Semantics of Discrete-Event Systems. Retrieved from https://bcourses.berkeley. edu/courses/1195544/files/folder/Lecture\%20Notes?preview=45232443.

[36] Edward Ashford Lee. 2017. Plato and the Nerd - The Creative Partnership of Humans and Technology. The MIT Press. 
[37] Edward A. Lee and Eleftherios Matsikoudis. 2009. The semantics of dataflow with firing. In From Semantics to Computer Science: Essays in Memory of Gilles Kahn, Gérard Huet, Gordon Plotkin, Jean-Jacques Lévy, and Yves Bertot (Eds.). Cambridge University Press.

[38] Edward A. Lee, Jan Reineke, and Michael Zimmer. 2017. Abstract PRET machines. In Proceedings of the IEEE Real-Time Systems Symposium (RTSS'17).

[39] Edward A. Lee and Sanjit A. Seshia. 2017. Introduction to Embedded Systems - A Cyber-Physical Systems Approach (2nd ed.). The MIT Press, Cambridge, MA. Retrieved from http://LeeSeshia.org.

[40] Edward A. Lee and Haiyang Zheng. 2007. Leveraging synchronous language principles for heterogeneous modeling and design of embedded systems. In Proceedings of the International Conference on Embedded Software (EMSOFT'07) ACM, 114-123.

[41] Xiaojun Liu and Edward A. Lee. 2008. CPO semantics of timed interactive actor networks. Theor. Comput. Sci. 409, 1 (2008), 110-125.

[42] Xiaojun Liu, Eleftherios Matsikoudis, and Edward A. Lee. 2006. Modeling timed concurrent systems. In CONCUR 2006 - Concurrency Theory, Vol. LNCS 4137. Springer, 1-15.

[43] Marten Lohstroh. 2020. Reactors: A Deterministic Model of Concurrent Computation for Reactive Systems. Ph.D. Dissertation. EECS Department, University of California, Berkeley. Retrieved from http://www2.eecs.berkeley.edu/Pubs/ TechRpts/2020/EECS-2020-235.html.

[44] Marten Lohstroh, Íñigo Íncer Romeo, Andrés Goens, Patricia Derler, Jeronimo Castrillon, Edward A. Lee, and Alberto Sangiovanni-Vincentelli. 2019. Reactors: A deterministic model for composable reactive systems. In Proceedings of the 8th International Workshop on Model-based Design of Cyber Physical Systems (CyPhy'19), Vol. LNCS 11971. SpringerVerlag.

[45] Marten Lohstroh, Martin Schoeberl, Andrés Goens, Armin Wasicek, Christopher Gill, Marjan Sirjani, and Edward A. Lee. 2019. Actors revisited for time-critical systems. In Proceedings of the 56th Design Automation Conference 2019 (DAC'19). ACM, 152:1-152:4.

[46] Oded Maler, Zohar Manna, and Amir Pnueli. 1992. From timed to hybrid systems. In Real-Time: Theory and Practice, REX Workshop. Springer-Verlag, 447-484.

[47] Louis Mandel, Cédric Pasteur, and Marc Pouzet. 2015. ReactiveML, ten years later. In Proceedings of the International Symposium on Principles and Practice of Declarative Programming (PPDP'15).

[48] Zohar Manna and Amir Pnueli. 1993. Verifying hybrid systems. In Hybrid Systems, Vol. LNCS 736. 4-35.

[49] Eleftherios Matsikoudis and Edward A. Lee. 2013. An axiomatization of the theory of generalized ultrametric semilattices of linear signals. In International Symposium on Fundamentals of Computation Theory (FCT), Vol. LNCS 8070. Springer, 248-258.

[50] Eleftherios Matsikoudis and Edward A. Lee. 2015. The fixed-point theory of strictly causal functions. Theor. Comput. Sci. 574 (2015), 39-77.

[51] Christian Menard, Andrés Goens, Marten Lohstroh, and Jeronimo Castrillon. 2020. Achieving derterminism in adaptive AUTOSAR. In Proceedings of the Design, Automation and Test in Europe Conference (DATE'20).

[52] Philipp Moritz, Robert Nishihara, Stephanie Wang, Alexey Tumanov, Richard Liaw, Eric Liang, William Paul, Michael I. Jordan, and Ion Stoica. 2017. Ray: A distributed framework for emerging AI applications. CoRR abs/1712.05889 (2017).

[53] Walid A. Najjar, Edward A. Lee, and Guang R. Gao. 1999. Advances in the dataflow computational model. Parallel Comput. 25, 13-14 (Dec. 1999), 1907-1929.

[54] NASA Engineering and Safety Center. 2011. National Highway Traffic Safety Administration Toyota Unintended Acceleration Investigation. Technical Assessment Report. NASA.

[55] Sibylla Priess-Crampe and Paulo Ribenboim. 1996. Generalized ultrametric spaces I. Abhand. Math. Semin. Univ. Hamb. 66 (1996), 55-73.

[56] Prokopec Aleksandar. 2018. Pluggable scheduling for the reactor programming model. In Programming with Actors: State-of-the-Art and Research Perspectives, Alessandro Ricci and Philipp Haller (Eds.). Springer International Publishing, 125-154.

[57] Aleksandar Prokopec and Martin Odersky. 2015. Isolates, channels, and event streams for composable distributed programming. In Proceedings of the ACM International Symposium on New Ideas, New Paradigms, and Reflections on Programming and Software (Onward!). ACM, New York, NY, 171-182.

[58] George M. Reed and A. W. Roscoe. 1988. Metric spaces as models for real-time concurrency. In Proceedings of the 3rd Workshop on Mathematical Foundations of Programming Language Semantics. 331-343.

[59] Raymond Roestenburg, Rob Bakker, and Rob Williams. 2016. Akka in Action. Manning Publications Co.

[60] Christian Schneider, Miro Spönemann, and Reinhard von Hanxleden. 2013. Just model! - Putting automatic synthesis of node-link-diagrams into practice. In Proceedings of the IEEE Symposium on Visual Languages and Human-centric Computing (VL/HCC’13), 75-82. 
[61] Martin Schoeberl, Wolfgang Puffitsch, Stefan Hepp, Benedikt Huber, and Daniel Prokesch. 2018. Patmos: A timepredictable microprocessor. Real-time Syst. 54, 2 (Apr. 2018), 389-423.

[62] Alexander Schulz-Rosengarten, Reinhard von Hanxleden, Frédéric Mallet, Robert de Simone, and Julien Deantoni. 2020. Time in SCCharts. In Languages, Design Methods, and Tools for Electronic System Design: Selected Contributions from FDL 2018, Tom J. Kazmierski, Sebastian Steinhorst, and Daniel Große (Eds.). Springer, 1-25. DOI : https://doi.org/ 10.1007/978-3-030-31585-6_1.

[63] Sanjit A. Seshia and Pramod Subramanyan. 2018. UCLID5: Integrating modeling, verification, synthesis and learning. In Proceedings of the 16th ACM/IEEE International Conference on Formal Methods and Models for System Design (MEMOCODE'18). IEEE, 1-10.

[64] Lui Sha, Abdullah Al-Nayeem, Mu Sun, José Meseguer, and Peter Ölveczky. 2009. PALS: Physically Asynchronous Logically Synchronous Systems. Technical Report. University of Illinois at Urbana Champaign (UIUC).

[65] B. D. Theelen, M. C. W. Geilen, T. Basten, J. P. M. Voeten, S.V. Gheorghita, and S. Stuijk. 2006. A scenario-aware data flow model for combined long-run average and worst-case performance analysis. In Proceedings of the International Conference on Formal Methods and Models for Co-design.

[66] Reinhard von Hanxleden. 2009. SyncCharts in C. Technical Report Bericht Nr. 0910. Department of Computer Science, Christian-Albrechts-Universitaet Kiel.

[67] Reinhard von Hanxleden et al. 2014. SCCharts: Sequentially constructive statecharts for safety-critical applications. In Proceedings of the ACM SIGPLAN Conference on Programming Language Design and Implementation (PLDI'14). ACM, New York, NY, 372-383.

[68] Reinhard Von Hanxleden, Timothy Bourke, and Alain Girault. 2017. Real-time ticks for synchronous programming. In Proceedings of the Forum on Specification and Design Languages (FDL'68). IEEE, 1-8.

[69] Reinhard Wilhelm et al. 2008. The worst-case execution-time problem-overview of methods and survey of tools. ACM Trans. Embed. Comput. Syst. 7, 3 (2008), 1-53.

[70] R. K. Yates. 1993. Networks of real-time processes. In Proceedings of the 4th International Conference on Concurrency Theory (CONCUR'93), E. Best (Ed.), Vol. LNCS 715. Springer-Verlag.

[71] Bernard Zeigler. 1976. Theory of Modeling and Simulation. Wiley Interscience, New York.

[72] Bernard P. Zeigler, Herbert Praehofer, and Tag Gon Kim. 2000. Theory of Modeling and Simulation (2nd ed.). Academic Press.

[73] Yang Zhao, Edward A. Lee, and Jie Liu. 2007. A programming model for time-synchronized distributed real-time systems. In Proceedings of the Real-time and Embedded Technology and Applications Symposium (RTAS'07). IEEE, 259268.

[74] Ye Zhou and Edward A. Lee. 2008. Causality interfaces for actor networks. ACM Trans. Embed. Comput. Syst. 7, 3 (2008), 1-35.

Received February 2020; revised October 2020; accepted January 2021 